\title{
Perspectivas de conservación desde múltiples actores: el caso de la Zona Ecológica de Preservación Chicamole, México
}

\author{
Perspectives of conservation from multiple actors: \\ the case of Chicamole Ecological Preservation Zone, Mexico
}

\author{
Karina Calva-Soto a, Jessica Bravo-Cadena ${ }^{\text {a }}$, Daniela Ortega-Meza a , María Teresa Pulido-Silva ${ }^{\text {a* }}$ \\ * Autor de correspondencia: a Universidad Autónoma del Estado de Hidalgo, \\ Centro de Investigaciones Biológicas, km 4,5 Carretera Pachuca-Tulancingo, Pachuca, Hidalgo, \\ Código Postal 42184, México, tel.: +52 771 3709711, mtpulido@yahoo.com
}

\begin{abstract}
SUMMARY
In Mexico, State Natural Protected Areas are frequently undervalued and rarely studied. The objective of this work was to analyze the process of decree and governance features of Chicamole Ecological Preservation Zone, which protects a cloud forest. An actor map was generated to understand the organization scheme, the external sector involved and their relationships. Semi structured Interviews were conducted both with governmental institutions and local inhabitants to analyze the decree. In spite of the obtained differences in perception, it can be noted that major conservation resulted from legislation. In addition, the presence of well-defined community norms regarding the use of natural resources (especially firewood and water) has encouraged forest conservation. This study shows the importance of a small area in the context of the protected areas in Mexico. The challenges of Chicamole Ecological Preservation Zone include increasing the interaction between sectors, achieving local recognition of the protected zone, and strengthening the social organization and the sustainable use of natural resources through stakeholder inclusion.
\end{abstract}

Key words: cloud forest, decision-making, governance.

\section{RESUMEN}

En México, las Áreas Naturales Protegidas Estatales son frecuentemente subvaloradas y rara vez estudiadas. El objetivo de este trabajo fue analizar el proceso de decreto y las características de gobernanza de la Zona de Preservación Ecológica Chicamole, que protege al Bosque Mesófilo de Montaña. Se generó un mapa de actores para conocer su esquema de organización y sus relaciones. Se aplicaron entrevistas semiestructuradas a las instituciones gubernamentales y a pobladores locales en pos de analizar el decreto. A pesar de que se obtuvieron diferencias en la percepción, se observa coincidencia respecto al efecto sobre la conservación a partir de la legislación. Además, la presencia de normas comunitarias bien definidas con respecto al uso de los recursos (sobre todo leña y agua), ha favorecido la conservación del bosque. Este estudio de caso mostró cómo esta pequeña área es importante en el contexto del sistema de Áreas Naturales Protegidas en México. Los retos de la Zona de Preservación Ecológica Chicamole incluyen incrementar la interacción entre los sectores, lograr su reconocimiento local y fortalecer la organización social y el aprovechamiento sostenible de los recursos, mediante la inclusión de actores.

Palabras clave: bosque mesófilo, gobernanza, tomadores de decisiones.

\section{INTRODUCCIÓN}

Existen diferentes estrategias de conservación biológica que pueden ser exitosas o fracasar dependiendo del sitio en donde se apliquen, los actores que intervienen y cómo se implementen. Una de ellas es la Conservación Basada en Comunidades, la cual busca que la gente local y otros actores participen en conjunto hacia objetivos comunes (Berkes y Seixas 2004). La Conservación Basada en Comunidades desarrolla redes de trabajo a diferentes niveles (del local al internacional) y en el que existen interrelaciones entre actores, tanto a un mismo nivel (relaciones horizontales) como entre niveles (verticales). De este modo, la toma de decisiones se logra mediante la comunicación directa entre partes, que facilitan el desarrollo de múltiples objetivos, en el que cada actor delimita sus necesidades y establece sus características de gobernanza (Barton y Merino 2004, Berkes y Seixas 2004, Alexander et al. 2016, Caballero et al. 2016). Casos exitosos de estrategias de Conservación Basada en Comunidades ya han sido repor- 
tados en diferentes partes del mundo, resaltando su importancia para mantener la biodiversidad (y sus servicios) y al mismo tiempo lograr el desarrollo de una región, utilizando como clave la interconexión entre actores (Berkes y Seixas 2004).

La relevancia de involucrar a la gente en la conservación biológica, es aun uno de los retos más importantes de conservación a nivel internacional. Esto se debe a que, al menos en algunas escalas, existe una sobreposición entre la distribución geográfica de la diversidad cultural y biológica (Posey y Overall 1990, Carlson y Maffi 2004), ratificando la importancia de incluir al hombre como elemento en la conservación biológica. En México, donde se calcula que el $26 \%$ del sistema de Áreas Naturales Protegidas es legalmente parte de los territorios de los pueblos indígenas (Boege 2008), es imperativo tomar en cuenta las necesidades y visiones de la gente local.

Las estrategias de conservación deben considerar la presencia humana y las particularidades de cada país. En México, derivado de su historia y características culturales, el $80 \%$ de las áreas boscosas son de propiedad colectiva, administradas como ejidos o tierras comunales, en donde también viven comunidades indígenas y habitantes con altos índices de marginación (Boege 2008, BezauryCreel y Gutiérrez 2009), las cuales tienen características específicas para su gobernanza. El éxito de las Áreas Naturales Protegidas y la Conservación Basada en Comunidades implica a la gobernanza en diferentes niveles y entre actores: individuos, instituciones, comunidades, empresas, Organizaciones no Gubernamentales, agencias internacionales, organizaciones religiosas y educativas. Estos actores pueden tener diferentes percepciones, intereses y preocupaciones sobre el uso de los recursos y de los objetivos de conservación (Borrini-Feyerabend et al. 2014, Alexander et al. 2016). De este modo, la gobernanza puede ayudar a maximizar los beneficios ecológicos, sociales, económicos y culturales de las Áreas Naturales Protegidas sin incurrir en costos innecesarios y disminuyendo los perjuicios (Borrini-Feyerabend et al. 2016).

Las Áreas Naturales Protegidas son la estrategia de conservación más ampliamente aplicadas, siendo una de los primeros mecanismos de conservación en diferentes partes del mundo, principalmente en países más desarrollados (Rivera y Vallejos-Romero 2015). En México, tienen un efecto directo en la conservación de la biodiversidad (Monroy et al. 2015), ocupando 24095694 ha del territorio nacional, existiendo 182 áreas de carácter federal, 421 estatales, 44 municipales, 24 ejidales y seis privadas. Las Áreas Naturales Protegidas estatales representan 3309 418 ha (Bezaury-Creel y Gutiérrez 2009). Mientras que las Áreas Naturales Protegidas estatales son una estrategia recurrente en México (presente en 22 de los 31 estados), su impacto es rara vez evaluado. En este estudio de caso se comparan y analizan las visiones de diversos actores locales y estatales acerca de la Zona de Preservación Ecológica Chicamole, que conserva Bosque Mesófilo de Montaña en
Hidalgo, México. Estudios previos sugieren que esta tiene una notable importancia biológica, cultural y para la subsistencia local (Galván 2014).

El Bosque Mesófilo de Montaña es prioritario en México e Hidalgo, debido a que su múltiple cantidad de microhábitats alberga el $10 \%$ de la diversidad biológica del país; sin embargo, se considera que hasta ahora se ha perdido casi la mitad de su cobertura original. Esto ha puesto en riesgo la biodiversidad que alberga y los servicios ecosistémicos que brinda. Los Bosques Mesófilos de Montaña del municipio San Bartolo Tutotepec, Hidalgo, se han caracterizado por su alta diversidad biológica y cultural sobre todo en los ejidos de Tuto y Pueblo Nuevo (antes Chicamole). En este último se encuentra la Zona de Preservación Ecológica Chicamole, el cual es un fragmento de bosque conservado, que presenta un manejo comunal, con población indígena y altos índices de marginación, por lo que surge la intención de evaluar cómo el establecimiento de esta Área Natural Protegida estatal y la gobernanza local han contribuido en el mantenimiento de este fragmento.

Este estudio de caso tiene como objetivo comparar las visiones de los diversos autores acerca de la presencia, uso y conservación de la Zona de Preservación Ecológica Chicamole, así como generar un mapa de relaciones entre estos. Debido, por una parte, a la localización relativamente remota del sitio que puede dificultar las intervenciones frecuentes de agentes externos $\mathrm{y}$, por otra, a la pequeña área que abarca, lo que le puede restar importancia, se plantea como hipótesis que existen visiones encontradas entre actores y que hay relativamente poca colaboración entre ellos hacia la conservación de esta zona. Este estudio de caso aporta información teórica y de campo acerca de las problemáticas y de la toma de decisiones de conservación en áreas pequeñas.

\section{MÉTODOS}

Contexto de la investigación y área de estudio. Los ejidos - que son una forma de propiedad colectiva de la tierra se establecieron en México como resultado de la Revolución Mexicana (ocurrida de 1910 a 1920), sin embargo, recibieron personalidad jurídica y patrimonio propio hasta 1992, a partir del decreto de la Ley Agraria (Trujillo 2009). Generalmente los territorios ejidales se dividen en asentamientos humano, tierras de uso común y tierras parceladas. En las tierras de uso común se desarrollan actividades comunales (Ostrom 2000, Barton y Merino 2004), mientras que en las áreas parceladas se desarrollan actividades económicas con beneficios individuales o familiares.

Todas las decisiones sobre el manejo y orden de estas zonas son tomadas por la asamblea (conformada por ejidatarios titulares de derechos ejidales, en su mayoría hombres), quienes desarrollan su propio reglamento; este es avalado por el Registro Agrario Nacional, órgano desconcentrado de la Secretaría de la Reforma Agraria. El ejido 
se conforma además por el comisariado ejidal y el consejo de vigilancia. En el ejido se pueden encontrar otros dos grupos de pobladores, "los posesionarios", quienes poseen título de tierras parceladas pero no tienen derecho a la tierra común y los "avecindados", quienes no tienen derechos sobre las tierras parceladas ni de uso común (SAGARPA 2012).

En México además, se han desarrollado otras estrategias de conservación, como los corredores biológicos, que "son un espacio geográfico delimitado que proporciona conectividad entre paisajes, ecosistemas y hábitats naturales o modificados y asegura el mantenimiento de la biodiversidad y los procesos ecológicos y evolutivos" (Ramírez 2003). Es el caso del Corredor Ecológico de la Sierra Madre Oriental, donde se busca generar la vinculación institucional y sectorial (CESMO 2016).
El estudio se llevó a cabo en la región Otomí-Tepehua del estado de Hidalgo, localizada en la Sierra Madre Oriental, en los municipios de Huehuetla (región de habla tepehua), San Bartolo Tutotepec y Tenango de Doria (zonas otomíes), donde convergen una alta riqueza biológica y cultural. Esta región es la tercera de importancia indígena en Hidalgo, donde existe una alta permanencia de rasgos de gobierno indígena, siendo una de las de mayor marginación y pobreza alimentaria del país (INEGI 2010) y alta riqueza biológica.

El estudio fue realizado en la Zona de Preservación Ecológica Chicamole, localizada dentro del Ejido de Chicamole, en la comunidad de Pueblo Nuevo, municipio de San Bartolo Tutotepec, Hidalgo (figura 1). Esta cuenta con una superficie de 67,56 hectáreas, mismas que corresponden a tierras de uso común, representando el $40 \%$ de la

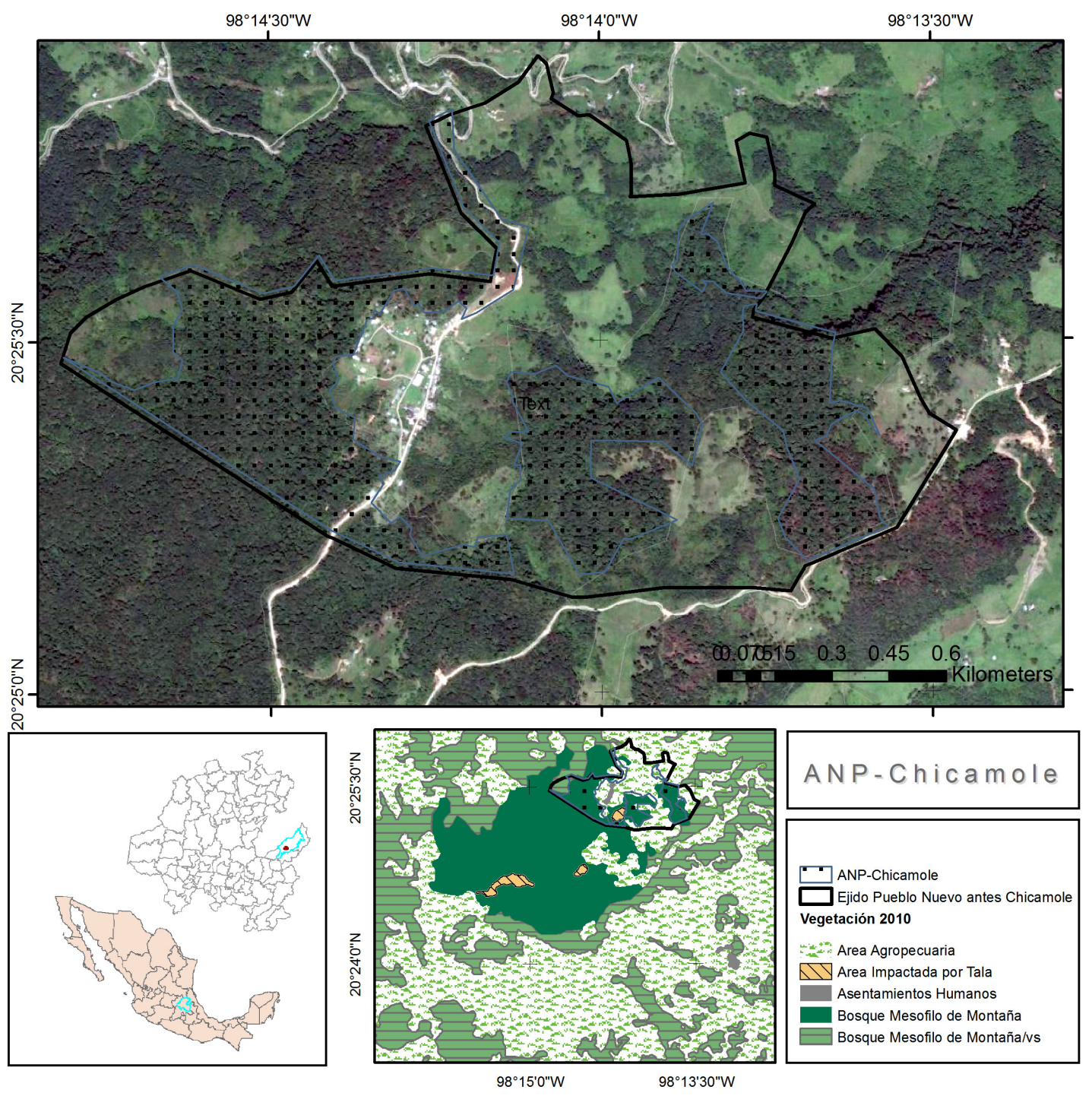

Figura 1. Ubicación del área de estudio. Se muestra el uso de suelo en 2010.

Location of the study area. Land use in 2010 is shown. 
superficie total del ejido (SEMARNATH 2016). La localidad se localiza a una altitud media de 1.840 m s.n.m. y una cumbre máxima de $2350 \mathrm{~m}$ s.n.m. La población total de Pueblo Nuevo en el 2010 era de 148 personas, 76 hombres y 72 mujeres (otomíes en su totalidad); así como 57 menores de edad y 91 adultos, con una mediana escolaridad de 3,96 años y $31 \%$ de la población económicamente activos (INEGI 2010).

Encuestas y análisis de datos. En septiembre del 2016 se realizaron once entrevistas semiestructuradas a diversos actores de la localidad, incluyendo a autoridades regionales (tres), ejidatarios (cuatro) y avecindados (cuatro). Las entrevistas se centraron en dos temáticas centrales: el establecimiento y gobernanza de la Zona de Preservación Ecológica Chicamole, así como el manejo de los recursos del bosque antes y a partir del establecimiento de esta. Lo anterior, se realizó un taller participativo en el que se desarrolló la línea del tiempo de la zona, a partir de la conjunción de los conocimientos de los pobladores sobre su comunidad, con el objetivo de conocer cuáles han sido los cambios significativos sobre el manejo de los recursos naturales y la creación del Área Natural Protegida (Geilfus 2002). Al mismo tiempo, esta información permitió diagramar un mapa de actores o diagrama de Venn (Geilfus 2002), que muestra las relaciones entre ellos y cómo ocurren los procesos de toma de decisiones a diversas escalas.

De modo complementario y con el fin de ampliar las perspectivas sobre la zona, se llevaron a cabo dos entrevistas semiestructuradas a personal de la Secretaría de Medio Ambiente y Recursos Naturales de Hidalgo y la Comisión Nacional de Áreas Naturales Protegidas. En ambos casos las preguntas se centraron en el establecimiento del Área Natural Protegida, sus características de gobernanza, así como sus visiones sobre las potencialidades y limitantes de la Zona de Preservación Ecológica Chicamole.

Para conocer los usos locales de los recursos del bosque se empleó la técnica de Lista Libre (Bernard 2006), entrevistando a la gente local, empleando las categorías locales de uso del bosque. Esta lista se analizó utilizando el Índice de Relevancia de Smith para determinar los resultados del Consenso Cultural por medio del programa ANTHROPAC (Borgatti 1996). Este índice otorga un valor numérico a cada uso mencionado, en función del orden de mención y de la cantidad de gente que refiere el uso. Saltos o discontinuidades numéricas en los valores resultantes permiten separar a un uso o subconjunto de usos más importantes de aquellos de menor importancia. Así, los mayores valores del índice se le dan a los elementos que tendieron a ser más referidos y nombrados en los primeros lugares de mención.

\section{RESULTADOS}

La iniciativa de la creación de La Zona de Preservación Ecológica Chicamole se la adjudican simultáneamente las autoridades locales y las instituciones gubernamentales. Áreas Naturales Protegidas: visión de las Secretarías. Secretaría de Medio Ambiente y Recursos Naturales de Hidalgo, quien fue la institución involucrada directamente en la conformación de la Zona de Preservación Ecológica Chicamole, percibe que dada la importancia del bosque mesófilo de montaña en la recarga de acuíferos y manantiales, así como la presión ejercida por la comunidad para el uso del área de uso común $(67,57 \mathrm{ha})$, las autoridades locales de la comunidad de Pueblo Nuevo se vieron en la necesidad de proteger su bosque, motivo por el cual, por iniciativa propia se acercaron a la institución, solicitando el establecimiento del Zona de Preservación Ecológica Chicamole.

En el año 2007 se iniciaron las gestiones para formalizar el área de preservación, con apoyo de Presidencia Municipal y ejidatarios, quienes participaron activamente en cada uno de los pasos. Las etapas desarrolladas fueron la solicitud por parte de la comunidad, la visita técnica realizada en la zona de interés, la elaboración del plan de manejo a consideración de las necesidades del ejido (incluyendo zonificación), el desarrollo del reglamento y su aprobación por la asamblea; la emisión del Certificado de Decreto e inscripción ante el Registro Agrario Nacional. La intención del decreto fue dar certeza jurídica al área para frenar la repartición de tierras.

A pesar de no llevarse a cabo monitoreos en la Zona de Preservación Ecológica Chicamole, a partir de la emisión del Decreto en 2008, la Secretaría indica que lleva a cabo reuniones regionales con el fin de identificar problemáticas y proponer acciones futuras. Además, a partir del establecimiento del Área Natural Protegida, la Secretaría de Medio Ambiente y Recursos Naturales de Hidalgo considera un aumento en los apoyos económicos a la zona, mediante el acceso a programas gubernamentales enfocados en la conservación. Estos han incluido: cercados perimetrales, letreros, acciones de educación ambiental y proyectos productivos orientados hacia la conservación, tales como módulos apícolas, Unidades de Manejo para la Conservación de la Vida Silvestre, proyectos ecoturísticos (senderos), restauración, brechas corta fuego y conservación de suelo. EEEEntre los retos que identifica la Secretaría de Medio Ambiente y Recursos Naturales de Hidalgo se encuentra el aumentar los beneficios económicos y culturales que obtienen los pobladores sobre sus recursos naturales, sin deteriorar la funcionalidad ecológica. Por otro lado, se resaltó la necesidad mantener la vigencia del decreto.

La Comisión Nacional de Áreas Naturales Protegidas, por su parte, considera que el interés de la creación de la Zona de Preservación Ecológica Chicamole surgió del Estado, como una estrategia de conservación hacia el Bosque Mesófilo de Montaña, siendo en menor medida, una iniciativa de los pobladores. Desde el punto de vista de la Comisión Nacional de Áreas Naturales Protegidas, la importancia de la Zona de Preservación Ecológica Chicamole radica en su localización dentro del Corredor Ecológico 
de la Sierra Madre Oriental y de la Región Prioritaria para la Conservación, lo que ha permitido que Secretaría de Medio Ambiente y Recursos Naturales de Hidalgo y la Federal integren intereses y trabajo en conjunto. Además, el sitio es de vital importancia, al formar parte de la Cuenca del Río Tuxpan, ya que provee de agua a varios municipios y por ser un sitio con alto patrimonio ecológico, cultural e histórico.

Se hace referencia a que la selección del polígono de la Zona de Preservación Ecológica Chicamole fue basada en el área del ejido, lo que trae ventajas y desventajas, ya que al ser los ejidatarios los únicos que toman decisiones en la asamblea y al estar este grupo conformado por personas de tercera edad, la gente joven, que no son ejidatarios, no se involucra y no tiene derecho a tomar decisiones o en la definición del territorio. Para el Corredor Ecológico de la Sierra Madre Oriental la delimitación de las zonas prioritarias para la conservación se llevan a cabo utilizando la conjunción del criterio social, ecológico y cultural, por lo que incluye una visión más integral; siendo clave al establecer modelos de trabajo que a largo plazo pueden ser replicados.

Se reconoce que muy pocas personas tienen conocimiento de esta zona y que a pesar de no participar en el proceso de decreto, la Comisión Nacional de Áreas Naturales Protegidas mantuvo una relación directa con la Zona de Preservación Ecológica Chicamole del año 2012 al 2016, periodo en el que se implementaron diferentes apoyos, tales como el Programa de Empleo Temporal, en el que se establecieron una brigada contra incendios, brechas cortafuegos y manejo de combustibles; así como el Programa de Conservación para el Desarrollo Sostenible, con la asignación de estufas ahorradoras de leña.

En este proceso, se logró un grupo de trabajo consolidado con la comunidad, diferentes universidades, el gobierno municipal e instituciones internacionales. Incluso durante ese periodo, con apoyo de la Comisión Nacional para el Desarrollo de los Pueblos Indígenas se inició un protocolo Biocultural, traducido al Otomí por jóvenes locales. La colaboración era tal que los casos de tala eran inmediatamente reportados al municipio y a la Comisión Nacional de Áreas Naturales Protegidas. Esta relación se ha mantenido el último año, aunque ha existido una menor colaboración debido a cambios gubernamentales y a pérdida de vinculación.

Finalmente se considera que uno de los principales retos de las Áreas Naturales Protegidas es el establecimiento de conexiones entre grupos de trabajo de zonas conservadas y áreas sin decreto, en las que se pierde el marco legal y se requieren otras estrategias de conservación, con mayor organización interna, por lo que se debe regularizar la posesión de tierras de los ejidos por parte de la Secretaría Agraria.

Áreas Naturales Protegidas: visión de la comunidad. De acuerdo a los pobladores de Pueblo Nuevo, han ocurrido diferentes acontecimientos que influyeron en el desarrollo de la comunidad, trayendo un aumento notorio en cuanto a infraestructura y servicios, que generaron un cambio en el uso de suelo y un aumento en los últimos nueve años en las actividades de conservación (cuadro 1).

Se observaron diferentes percepciones con respecto a la visión sobre esta zona por parte de los habitantes. Mientras que todos los pobladores del ejido ubican al bosque como un área de uso común, en donde los recursos naturales no se pueden extraer, solo tres personas asocian esa área con lo que se conoce como la Zona de Preservación Ecológica Chicamole. Los avecindados no la reconocen o no han escuchado siquiera de ella, reconociendo que no se puede extraer madera. En contraste, los ejidatarios muestran percepciones diversas: 1) dos no han escuchado sobre el establecimiento del Área Natural Protegida, aunque saben de las reglas de uso de los recursos; 2) otros dos identifican el área, sin embargo, la confunden con la Unidad de Manejo para la Conservación de la Vida Silvestre; 3) uno sabe del establecimiento del área, pero lo percibe como un área que fue otorgada al gobierno para su protección, por lo que no se permite el uso de los recursos de esta zona; 4) por último, sólo una persona reconoce el área como un Área Natural Protegida; sin embargo, se debe resaltar que esta persona fue el representante del ejido involucrado en el proceso administrativo de formalización del Área Natural Protegida. Por último, de las autoridades locales actuales, uno desconoce el decreto del área, otro reconoce que se inició el proceso, pero entiende que nunca se obtuvo una resolución por parte de Secretaría de Medio Ambiente y Recursos Naturales de Hidalgo, otro más la ubica como una zona de preservación. Es importante mencionar que aquellos individuos que reconocen la Zona de Preservación Ecológica Chicamole, son o fueron comisariados ejidales.

Las autoridades locales coinciden en la fecha de inicio del proceso del decreto entre los años 2008 y 2009. Además, confirman que los ejidatarios son los únicos que pueden participar en las reuniones de asamblea, por lo que fueron la única parte de la comunidad que participaron en el desarrollo del decreto. Con respecto a los actores externos que colaboraron en el proceso, reconocen a instituciones gubernamentales, como la Secretaría de Medio Ambiente y Recursos Naturales (federal y estatal), la Comisión Nacional de Áreas Naturales Protegidas y la Comisión Nacional para el Desarrollo de los Pueblos Indígenas, así como a técnicos.

La mayoría reconocen la iniciativa del establecimiento de la Zona de Preservación Ecológica Chicamole como proveniente de externos, aunque no existe un consenso sobre quien lo inició, teniendo diferentes visiones: 1) Secretaría de Medio Ambiente y Recursos Naturales Federal, a través de sus técnicos, 2) fue resultado de una propuesta de proyecto para el establecimiento de una Unidad de Manejo para la Conservación de la Vida Silvestre, 3) fue resultado de una invitación a un curso y propuesta de proyecto, 4) solo una persona menciona que el Comisariado (en aquel 
Cuadro 1. Línea de tiempo desarrollada por los comunitarios de Pueblo Nuevo.

Timeline developed by the community of Pueblo Nuevo.

\begin{tabular}{|c|c|}
\hline Año* & Evento \\
\hline 1936 & Inicio de la comunidad \\
\hline 1951 & Comenzó a poblarse, migración de la comunidad de Chicamole a Pueblo Nuevo \\
\hline 1966 & Construcción de la primera escuela, llegada de soldados que enseñaban a leer y escribir \\
\hline 1976 & Emigración por trabajo \\
\hline 1976/1989 & Creación de la escuela nueva \\
\hline 1990 & Daños a la cosecha por el huracán Diana \\
\hline 1991 & Creación de la carretera \\
\hline 1993 & Creación de la clínica de salud \\
\hline 1993 & Se implementó el servicio eléctrico en el sitio \\
\hline 1997 & Parcelaron sus tierras por parte del gobierno \\
\hline 2000 y 2005 & Se entubó el agua de dos manantiales \\
\hline 2006 & Cambio de forma de cultivo \\
\hline 2006 & Se requiere el permiso del presidente del comisariado para extraer leña del bosque \\
\hline 2007 & Inicio del Decreto de la Reserva Ecológica el Chicamole \\
\hline 2010 & Entró el proyecto de las brechas cortafuego \\
\hline 2011 & Se regularizó el consumo de leña por tarea \\
\hline 2013 & Ocurrieron sequía más fuerte que otros años \\
\hline 2013 & Se establecen huertos de hortalizas \\
\hline 2014 & Se establece la Unidad de Manejo para la Conservación de la Vida Silvestre del Temazate y la construcción de una cabaña \\
\hline 2015 & Un incendio afectó las comunidades del Hongo y el Encinar \\
\hline
\end{tabular}

*2007-2015: período en que ha existido la zona de Protección Ecológica Chicamole.

turno) tuvo la iniciativa de llevar el proyecto a las oficinas de Secretaría de Medio Ambiente y Recursos Naturales de Hidalgo.

La mayoría coincide en que el área se desarrolló para beneficiar a la comunidad con la obtención de recursos económicos para la generación de proyectos productivos. Solo una persona la mencionó como como una estrategia para cuidar el agua y el oxígeno.

Pocos identifican los límites de la Zona de Preservación Ecológica Chicamole, confundiendo el área con la Unidad de Manejo para la Conservación de la Vida Silvestre para venado temazate (Mazama temama (Kerr, 1792)) que se estableció en la zona. Otro reconoce la localización del área, pero no su extensión, pues solo mencionan 8 ha, lo que contrasta con las 67 ha que fueron decretadas. De manera general, toda la gente identifica a la Zona de Preservación Ecológica Chicamole con un área no parcelada de uso común, llamada localmente "vacante", la cual fue declarada por el Programa de Certificación de Derechos Ejidales y Titulación de Solares, áreas para asentamientos humanos y áreas de producción agrícola.
Acuerdos internos sobre el uso de los recursos de bosque. A pesar de que el decreto de la Zona de Preservación Ecológica Chicamole no es claro para todos los habitantes y únicamente dos ejidatarios tienen conocimiento de la presencia de un reglamento interno, existen reglas sobre el manejo de los recursos que son reconocidas y aplicadas tanto por avecindados como por ejidatarios. Estas normas se han desarrollado en el contexto de su forma tradicional del uso de recursos. Las más reconocidas son la prohibición de tirar basura, talar árboles y cazar. La leña del área común, únicamente es para uso doméstico de los avecindados y ejidatarios, y solo se puede obtener de material presente en el suelo o mediante la tala de "palo seco" (árboles muertos). Además, se lleva un control de cuánta leña se le autoriza a cada familia. Está prohibido tirar "palo verde" (árboles vivos en pie), a excepción de aquellos que requieran construir sus casas, los cuales deben solicitar autorización al Presidente del Comisariado Ejidal, quien evalúa las condiciones de la familia y otorga un oficio sellado y firmado. En estos casos le corresponde al presidente de comité de vigilancia verificar in situ qué árbol se va a de- 
rribar y no hay mecanismos externos de verificación. $\mathrm{La}$ venta de madera está prohibida.

Reconocen diferentes sanciones para la tala ilegal de madera, por ejemplo, para cada árbol tirado, se deben de plantar entre tres y diez árboles y cuando llegan apoyos de gobierno para reforestación, todos deben participar en la faena (sin embargo, esto no siempre se cumple). Además se mencionó que la policía hace recorridos de vigilancia en la zona dos o tres veces a la semana, deteniendo a quien sorprenda talando. Otros consideran que en realidad no hay vigilancia ni sanciones a pesar de que existe vigilancia por parte del municipio. La mayoría menciona que estas normas y sus sanciones vienen de órdenes de San Bartolo Tutotepec (cabecera municipal) y Pachuca (capital del estado).

A pesar de no reconocer a la Zona de Preservación Ecológica Chicamole, los habitantes de la comunidad observan cambios en su forma de vida en los últimos seis o siete años. Mencionan que previamente se podía talar, mientras que ahora ya existe un control, lo que ha llevado a que la gente aproveche el recurso de forma clandestina, reduciendo la cobertura del bosque. Con respecto a la caza, a pesar de que siempre se ha prohibido, se sigue realizando, principalmente de armadillo, temazate, tuza real y tejón.

Importancia del bosque. Los habitantes de la comunidad reconocen al bosque por ser proveedor de aire y agua, pero sobre todo de leña y de madera. También lo identifican como hábitat de diversas especies vegetales y animales, así como por brindarles un bello paisaje que observar. Además, lo consideran indispensable para que sus futuras generaciones tengan dónde vivir.
A partir del análisis de la lista libre fue posible identificar que existen 12 usos de los recursos del bosque por parte de las habitantes de la comunidad de Pueblo Nuevo, categorizados en sus propios términos. El uso de la leña fue el más citado (100\%), seguido por los hongos, la madera y el agua (cuadro 2). Es importante notar que ellos separaron a los hongos de otros alimentos, debido a la importancia de los hongos per se.

Con el uso del Índice de Smith fue posible identificar tres rupturas en la lista libre, de modo que en la primera ruptura el uso de la 'leña' presentó el valor más elevado $(0,842)$. La segunda incluye el uso de hongos, agua y madera. Finalmente en la última ruptura se identifican los usos con una mención menor y menor importancia dada por lo habitantes (cuadro 2).

Con respecto a la leña, se observó una proporción variable en su uso entre las familias. Se reportan hogares donde únicamente se utiliza una tarea (amontonamiento de leña de $80 \mathrm{~m}$ de largo x 3,20 m de alto) al mes. Otros utilizan una tarea cada dos o tres meses. Tres casos reportan el uso de una tarea en periodos de cuatro a seis meses. Además, resalta que en predios particulares no se cuantifica el uso de este recurso, ni se solicita permiso. Las autoridades confirmaron que se otorgan de cuatro a cinco tareas por familia al año y que en total en el ejido cada dos meses se extraen aproximadamente 10 árboles.

Los pobladores identifican al agua como otro recurso indispensable, con el cual tienen una relación cultural importante, e identifican al bosque como fuente de la misma. Mencionan que previamente se realizaban ofrendas a los cuerpos de agua, ritual en el que una procesión llevaba flo-

Cuadro 2. Frecuencia de citas del uso de los recursos del bosque en Chicamole. Se emplearon las categorías locales de uso de recursos: "alimento" es fauna y flora silvestre; "hongos" son comestibles; "plantas": incluye hierbas medicinales.

Frequency of citations of the use of forest resources. Local categories of resource use were used: "alimento" (food) is wild fauna and flora; "hongos" (mushrooms) are edible; "plantas" (plants) includes medicinal herbs.

\begin{tabular}{lccc}
\hline Recurso & Frecuencia (\%) & Promedio & Índice de relevancia de Smith \\
\hline Leña & 100,0 & 1,55 & 0,842 \\
Hongos & 36,4 & 1,75 & 0,242 \\
Agua & 18,2 & 1,00 & 0,182 \\
Madera & 27,3 & 3,00 & 0,109 \\
Tierra & 9,1 & 1,00 & 0,091 \\
Aire limpio & 9,1 & 2,00 & 0,073 \\
Plantas & 9,1 & 2,00 & 0,068 \\
Siembra & 9,1 & 2,00 & 0,045 \\
Refugio de animales & 9,1 & 4,00 & 0,036 \\
Alimento & 9,1 & 3,00 & 0,030 \\
Milpa & 9,1 & 3,00 & 0,030 \\
Trabajo & 9,1 & 4,00 & 0,023 \\
\hline
\end{tabular}


res a la laguna y al manantial. En sus palabras 'esta costumbre se realiza en tiempo de sequía para que regrese el agua'. Sin embargo, actualmente se lleva a cabo con poca frecuencia por falta de recursos económicos. También se ejecutan ofrendas a ciertos árboles grandes para pedir que no haya problemas en la comunidad, en períodos aproximados de dos años. Conjuntamente, el bosque impacta en su expresión artesanal, ya que en los "tenangos" (bordados típicos de la región) se hacen representaciones fantasiosas de flora y fauna.

Percepción de apoyos gubernamentales. La mayoría identifica un incremento en los últimos años, tales como el proyecto de Programa Estratégico de Seguridad Alimentaria con gallineros y con huertos de hortalizas, la Unidad de Manejo para la Conservación de la Vida Silvestre de la tuza real (Cuniculus paca (Linnaeus, 1766)) y la de temazate, que trajo el establecimiento de una cabaña, senderos turísticos, un puente de acceso al bosque, áreas circuladas y torres de observación. Esto aumentó el turismo, de modo que desde hace casi dos años la comunidad es visitada para realizar actividades como caminata, senderismo y observación de flora y fauna, proporcionando un ingreso monetario bajo.

Percepción de comunidades vecinas y otros usos del territorio. Las personas de la comunidad $(81 \%)$ han observado una diferencia en la conservación de su bosque comparado con los sitios vecinos. En comparación con Tuto, el $36 \%$ percibe que aquel tiene el doble de la extensión del terreno lo que permite un mayor uso de recursos maderables de modo comercial (Pinus L. y Quercus L.), así como la modificación del tipo de suelo para el establecimiento de potreros $(9 \%)$. Por lo anterior, la percepción de los habitantes es que hay más tala y no respetan el bosque como se hace en la Zona de Preservación Ecológica Chicamole, el cual se encuentra mejor conservado (27\%). El $9 \%$ considera que el bosque de Chicamole sería más beneficioso si se permitiera la construcción, la agricultura y la ganadería.

Con respecto a la agricultura, cada habitante de la comunidad tiene un área para sembrar (aproximadamente 2 ha), sin embargo, este no se aprovecha por falta de recursos económicos. A pesar de esto, cuando hay posibilidad, se cultiva frijol, maíz y cempasúchil. En los huertos familiares se tiene aguacate, calabaza, chayote y hortalizas, principalmente para autoconsumo. Todos coinciden en que por el clima y las condiciones del terreno es muy complicado mantener ganado bovino, por lo que solo identifican a una familia dedicada a esta actividad (en pequeña escala). Algunos poseen animales de corral como pollos, guajolotes, puercos y borregos.

Otras actividades, como el ecoturismo, se han planteado como opciones alternativas al aprovechamiento del bosque para generar fuentes de trabajo; sin embargo, los beneficios económicos que se perciben en la actualidad no sitúan a esta actividad como una alternativa en compara- ción con los ingresos que se podrían obtener del manejo forestal. Su relevancia monetaria es baja y solo una de las personas lo mencionó.

Mapa de actores. En la Zona de Preservación Ecológica Chicamole, con 67 ha, intervienen múltiples actores, que van desde la escala internacional, nacional, estatal, municipal y local, siendo más numerosos los últimos (figura 2). Las relaciones identificadas, representan vinculaciones de los últimos seis años, por lo cual en el momento actual pueden no estar conectados o relacionados todos los actores o bien pueden no encontrarse algunos vínculos. Mientras que existen interacciones horizontales al mismo nivel de trabajo, las relaciones entre niveles verticales son menos recíprocas. Algunas de estas relaciones están más claramente definidas que otras (figura 2).

\section{DISCUSIÓN}

Este estudio de caso muestra claramente la gran cantidad de actores que existen desde el nivel local al internacional, involucrados en el uso y conservación de un área de tan solo 67 ha, como es el caso de Zona de Preservación Ecológica Chicamole. El interés de la Comisión Nacional de Áreas Naturales Protegidas por realizar acciones piloto en el ejido (del 2011 al 2015) generó presencia institucional, la cual fue percibida por los habitantes de manera cercana, con mayor fortaleza que otras instituciones. Además, esta Comisión logró la incorporación de actores internacionales. Para que este u otros proyectos de conservación sean exitosos es indispensable la comunicación entre los distintos niveles, de modo que exista un aprendizaje mutuo entre actores (Berkes y Seixas 2004). Por lo tanto, el reto es desarrollar estrategias de colaboración vertical de los niveles locales a los estatales y nacionales que implementen objetivos con beneficios en común, al mismo tiempo que se mantengan y fortalezcan las relaciones horizontales ya existentes.

Uno de los pilares de la conservación basada en comunidades es la articulación de actores, mediante objetivos en común, que satisfagan sus diversas expectativas y necesidades, al mismo tiempo que permitan la conservación (Ostrom 2000, Berkes y Seixas 2004, Caballero et al. 2016). En esta zona la mayor parte de los actores involucrados comparten el mismo objetivo de mantener el bosque, aunque las motivaciones no siempre se basen en un interés conservacionista per se. La realidad observada es que este fragmento de bosque se mantiene en buen estado de conservación por diversas causas. Los indígenas otomíes cuidan su bosque por razones más idiosincráticas y prácticas que por la propia visión de los comunitarios de proteger ("visión conservacionista"), ya que como algunos mencionaron, sería mejor poder usar esa tierra para la obtención de mayores ganancias económicas. Las razones idiosincráticas que llevan a que los otomíes contribuyan a la conservación incluyen, entre otros: a) los medios vida de los otomíes his- 


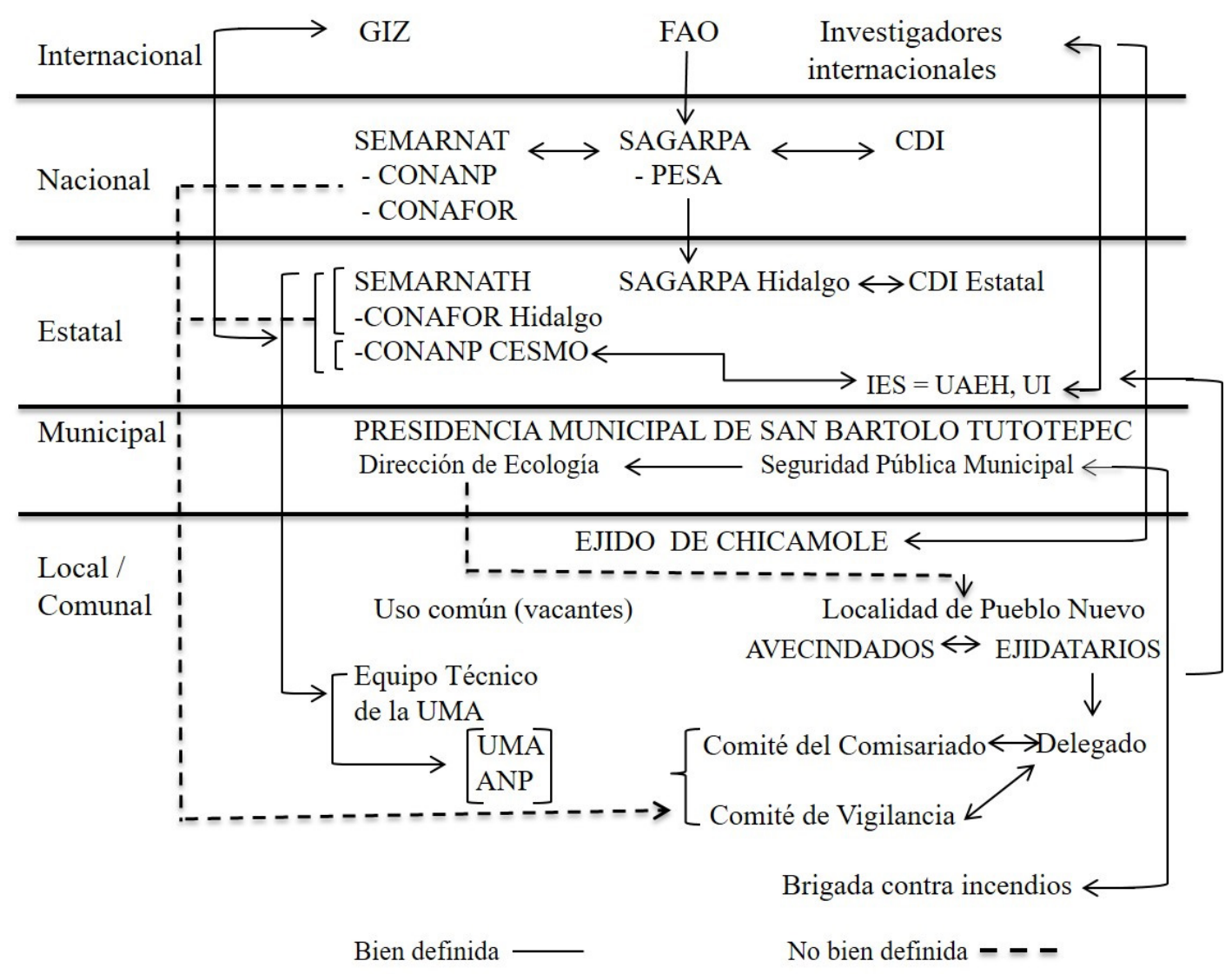

Figura 2. Esquema organizativo comunitario de la Zona de Preservación Ecológica Chicamole y su relación con agentes externos. Las flechas representan las interrelaciones entre sectores al mismo nivel (horizontales) y entre niveles (verticales), así como la dirección en la toma de decisiones. ANP: Área Natural Protegida; CESMO: Corredor Ecológico de la Sierra Madre Oriental; CDI: La Comisión Nacional para el Desarrollo de los Pueblos Indígenas; CONAFOR: Comisión Nacional Forestal; CONANP: Comisión Nacional de Áreas Naturales Protegidas; FAO: Organización de las Naciones Unidas para la Alimentación; GIZ: Deutsche Gesellschaft für Internationale Zusammenarbeit; IES: Instituciones de Educación Superior; PESA: Proyecto Estratégico de Seguridad Alimentaria; SAGARPA: Secretaría de Agricultura y Desarrollo Rural; SEMARNAT: Secretaría de Medio Ambiente y Recursos Naturales; SEMARNATH: Secretaría de Medio Ambiente y Recursos Naturales de Hidalgo; UAEH: Universidad Autónoma del Estado de Hidalgo; UMA: Unidades de Manejo para la Conservación de la Vida Silvestre; UT: Universidades Tecnológicas, entre ellas la Universidad Tecnológica de la Sierra Hidalguense.

Community organizational scheme of Chicamole Ecological Reserve and its relationship with external agents. The arrows represent the interrelations between sectors at the same level (horizontal) and between levels (vertical), as well as the direction in the decision making.

tóricamente no han incluido a la ganadería, siendo pueblos dependientes de matorrales y bosques mediante cacería y recolección; b) la presencia de un sistema de gobernanza tradicional sólido, respetado y con reglas bien establecidas sobre el manejo de los recursos, en especial la leña, ha permitido la regulación de la tala; c) la alta migración de jóvenes debido a la imposibilidad de hacer una vida allí, reduce la presión hacia el bosque, aunque implica costos sociales y económicos evidentes; d) su interés de mantener recursos vitales y espirituales que les brinda el bosque.

En contraste, las autoridades ambientales tienen como metas de trabajo el ayudar a conservar el bosque, mediante apoyos gubernamentales, como el turismo (cabaña y sendero), sin conocer aún su efecto sobre actividades extractivas. Es relevante realizar estudios sobre cuáles son las especies de hongos utilizadas y su aprovechamiento, así como estudiar otros recursos no maderables. Otra de las claves de la conservación basada en comunidades es que se logren satisfacer varios objetivos simultáneamente y que existan reglas claras y mecanismos de control que permitan una coordinación armoniosa entre los autores, en un escenario de "ganar-ganar" (Ostrom 2000). En este sentido, los objetivos locales de cubrir sus necesidades de autosubsistencia a partir de sus ecosistemas se ven contrapuestos a la visión conservacionista a ultranza de conservar mediante el desuso de los recursos. El conjunto de 
resultados obtenidos estuvieron acordes con la hipótesis, puesto que se encontró que las visiones e intereses de los actores frecuentemente se contraponen y que hay una incomunicación marcada, especialmente entre actores que están a distinta escala. Aún así, como se explicó, la mayoría comparten el objetivo común de mantener el bosque. Además, hay actores clave, como el caso de los funcionarios de la Comisión Nacional de Áreas Naturales Protegidas, que a pesar que son sólo dos personas, han servido como catalizadores de procesos en pro de la conservación y han buscado proactivamente la vinculación de actores a diversas escalas.

Importancia de la conservación de pequeñas áreas. El estudio de caso de la Zona de Preservación Ecológica Chicamole aporta evidencia clara de la importancia de estas 67 ha en varios sentidos: a) es una fuente directa e importante de recursos naturales para la comunidad, quienes se benefician directamente de ellos al ser utilizadas para autoconsumo y como una fuente de ingresos; $b$ ) por su pequeño tamaño brinda una mayor operatividad al tener un manejo adecuado del espacio y podría permitir una mayor comunicación entre actores (aunque esto último aún no ocurre completamente); c) contribuye al manejo integrado del paisaje. Por lo tanto, se propone tomar en cuenta casos semejantes no solo en México, sino en diferentes partes del mundo, de modo que pueda maximizarse su papel en la conservación biológica y desarrollo social.

Las estrategias de protección de la biodiversidad en México son diversas y se fortalecen por los mecanismos locales y regionales de conservación como esta, que toman relevancia dentro de un portafolio mayor de estrategias de mayor extensión territorial, lo que incluye al Corredor Ecológico de la Sierra Madre Oriental y a las Áreas Naturales Protegidas estatales, municipales y federales. En Hidalgo hay 46 Áreas Naturales Protegidas estatales de pequeño tamaño y cuatro federales, por lo que la implementación de otras estrategias de conservación facilita la conectividad, incrementando el efecto de las Áreas Naturales Protegidas. La conservación privada (en Chile), las áreas apoyadas con pagos por servicios ambientales y las áreas voluntarias de conservación (Oaxaca, México), han demostrado ser estrategias complementarias, que apoyan a la conectividad del paisaje y conservación de la biodiversidad, por lo lo que se proponen como estrategias complementarias de conservación para el área de estudio (Monroy et al. 2015, Rivera y Vallejos-Romero 2015).

Retos y recomendaciones. Uno de los principales retos es fomentar los vínculos e intercambio de información entre la Secretaría de Medio Ambiente y Recursos Naturales de Hidalgo, población local, Universidades, agencias de desarrollo, etc., para que se logre con más eficiencia la conservación del patrimonio biocultural de la zona. Ejemplo de esto incluye desarrollar investigación básica y aplicada, realizar apoyos de gobierno enfocados en la conservación y que se articulen con mayor eficiencia con iniciativas como el Corredor Ecológico de la Sierra Madre Oriental o estrategias de actividades productivas sustentables, las cuales generarían alternativas de empleo para disminuir la alta marginación y migración en la zona.

Otro reto importante es hacer un trabajo con la población local para que reconozcan a la Zona de Preservación Ecológica Chicamole en la dimensión legal, y así puedan cumplir y aprovechar de mejor forma sus derechos y obligaciones. Igualmente se sugiere el fortalecimiento de instituciones locales. Por ejemplo, la Asamblea (principal institución), está constituida mayoritariamente por personas de la tercera edad, que frecuentemente hablan solo otomí, lo que constituye una limitación para el acceso a información, pues en México el idioma dominante es el español. Se recomienda la participación de los jóvenes en estas Asambleas. Por esto, existen dos posibles escenarios, la decisión de la formación de un área protegida con el consenso en común de ejidatarios adultos que buscan la protección de la zona, o el apoyo de los ejidatarios con un conocimiento limitado de lo que se discutía en las asambleas. Esta inequidad se refleja en una situación social y económica desigual, en la cual las poblaciones más afectadas son aquellas que se encuentran en áreas rurales, que en su mayoría pertenecen a grupos indígenas (Monterroso 2006).

La debilidad operativa de las instituciones gubernamentales que manejan los recursos desde niveles centrales, la falta de recurso humano para el desarrollo de sus actividades (Berkes y Seixas 2004), son otros de los retos importantes por afrontar.

\section{CONCLUSIONES}

Este estudio muestra que el mantenimiento de pequeñas áreas de conservación es relevante y puede involucrar a un gran número de actores a distintas escalas. Las razones por las que estos actores conservan el bosque pueden ser idiosincráticas, a veces como respuesta a apoyos paternalistas, y en pocos casos su argumento es la conservación per se. Fue evidenciado que el talón de Aquiles incluye a las visiones encontradas entre actores, así como a la incomunicación y escasa colaboración entre estos. Los elementos más claramente identificados, que ayudarían al mantenimiento de ese bosque desde el punto de vista del marco conceptual de la conservación basada en comunidades, es lograr simultáneamente múltiples objetivos que satisfagan los intereses y expectativas de los diversos actores, tener reglas claras de cómo manejar esos recursos, conocer y construir a partir de las normativas propias de los grupos locales y, sobre todo, lograr vínculos verticales reales entre actores de diversos niveles. En este sentido, el mapa de actores generado y el entendimiento de las visiones y objetivos de los principales actores - que es lo que este estudio de caso aporta a nivel metodológico y de información son elementos indispensables para acercarse a la meta de la conservación. Uno de los aportes de actores externos, 
como lo son las universidades regionales, es precisamente el análisis teórico mostrado en este estudio de caso.

\section{AGRADECIMIENTOS}

Agradecemos a la comunidad de Pueblo Nuevo de San Bartolo Tutotepec, a las autoridades de Secretaría de Medio Ambiente y Recursos Naturales de Hidalgo y la Comisión Nacional de Áreas Naturales Protegidas por su valioso apoyo en las entrevistas. Se agradece también al Biol. Alejandro López-Portillo Vargas, al Ing. Ismael Alvarado Aguilar y a Juan Carlo García Lopena por su colaboración en el desarrollo de este estudio.

\section{REFERENCIAS}

Alexander S, M Andrachuk, D Armitage. 2016. Navigating governance networks for community-based conservation. Frontiers in Ecology and the Environment 14 (3): 155-164. DOI: https://doi.org/10.1002/fee.1251

Barton D, L Merino. 2004. La experiencia de la comunidades forestales en México. México DF, México. Instituto Nacional de Ecología (INE-SEMARNAT), Consejo Civil Mexicano para la Silvicultura Sostenible A.C. 269 p.

Berkes F, CS Seixas. 2004. Lessons from community self-organization and cross-scale linkages in four Equator Initiative Projects. Winnipeg, Canadá. CCBRM, University of Manitoba. $30 \mathrm{p}$.

Bernard RH. 2006. Research methods in Anthropology: qualitative and social mechanisms for build quantitative approaches. New York, USA. Altamira Press. 803 p.

Bezaury-Creel J, D Gutiérrez. 2009. Áreas naturales protegidas y desarrollo social en México. In Dirzo R, R González, IJ March eds. Capital Natural de México, vol. II, Estado de conservación y tendencias de cambio. México DF, México. CONABIO. p. 385-431.

Boege E. 2008. El patrimonio biocultural de los pueblos indígenas de México. Hacia la conservación in situ de la biodiversidad y agrobiodiversidad en los territorios indígenas. México DF, México. Instituto Nacional de Antropología e Historia - Comisión Nacional para el Desarrollo de los Pueblos Indígenas. $344 \mathrm{p}$.

Borgatti SP. 1996. ANTHROPAC 4.0. Analytic Technologies. Consultado 15 dic. 2016. Disponible en http://www. analytictech.com/anthropac/anthropac.htm

Borrini-Feyerabend G, N Dudley, T Jaeger, B Lassen, BN Pathak, A Phillips, T Sandwith. 2014. Gobernanza de áreas protegidas: de la comprensión a la acción. No. 20 de la Serie Directrices para buenas prácticas en áreas protegidas. Gland, Suiza. UICN. 123 p.

Caballero P, G Herrera, C Berriozabal, MT Pulido. 2016. Conservación basada en comunidad: importancia y perspectivas para Latinoamérica. Estudios Sociales 26(48): 335-352.

Carlson T J, L Maffi. 2004. Ethnobotany and conservation of biocultural diversity. New York, USA. New York Botanical Garden. 356 p. DOI: https://doi.org/10.1525/aa.2005.107.3.512

CESMO (Corredor Ecológico Sierra Madre Oriental, MX). 2016. ¿Qué es el CESMO? Consultado 11 ene. 2017. Disponible en: http://cesmo.zohosites.com/que-es-el-cesmo.html

Galván J. 2014. Potencial de una zona de bosque mesófilo de montaña en San Bartolo Tutotepec, Hidalgo para ser incorporada en el programa REDD+. Tesis de Maestría. Pachuca, México. Universidad Autónoma del Estado de Hidalgo. 81 p.

Geilfus F. 2002. 80 herramientas para el desarrollo participativo: diagnóstico, planificación, monitoreo, evaluación. San José, Costa Rica. IICA. 208 p.

INEGI (Instituto Nacional de Estadística y Geografía, MX). 2010. Consensos y Conteos. Censo de Población y Vivienda 2010 Principales resultados por localidad (ITER). Consultado 09 dic. 2016. Disponible en: http://www.inegi.org. $\mathrm{mx} /$ sistemas/consulta resultados/iter2010.aspx

Monroy AG, V Sánchez-Cordero, M Briones-Salas, R Lira-Saade, JM Maass Moreno. 2015. Representatividad de los tipos de vegetación en distintas iniciativas de conservación en Oaxaca, México. Bosque 36(2): 199-210. DOI: https://doi. org/10.4067/S0717-92002015000200006

Monterroso I. 2006. Comunidades locales en áreas protegidas: Reflexiones sobre las políticas de conservación en la reserva de Biosfera Maya. In Alimonda $\mathrm{H}$ ed. Los tormentos de la materia. Aportes para una ecología política latinoamericana. Buenos Aires, Argentina. CLACSO. p. 239-274.

Ostrom E. 2000. El gobierno de los bienes comunes. La evolución de las instituciones de la acción colectiva. México DF, México. UNAM/CFE. 403 p.

Posey DA, W Overall. 1990. Ethnobiology: Implications and Applications. Proceedings of the First International Congress of Ethnobiology. Belem, Brasil. Museu Paraense Emilio Goeldi. 363 p.

Ramírez D. 2003. El corredor Biológico Mesoamericano. CONABIO. Biodiversitas 47: 1-3.

Rivera C, A Vallejos-Romero. 2015. La privatización de la conservación en Chile: repensando la gobernanza ambiental. Bosque 36(1): 15-25. DOI: https://doi.org/10.4067/ S0717-92002015000100003

SAGARPA (Secretaría de Agricultura, Ganadería, Desarrollo Rural, Pesca y Alimentación, MX). 2012. Ley Agraria. Diario Oficial de la Federación, 09 de abril del 2012.

SEMARNATH (Secretaría de Medio Ambiente y Recursos Naturales de Hidalgo, MX). 2016. Áreas Naturales Protegidas del Estado de Hidalgo. Consultado 15 dic. 2016. Disponible en: http://conacyt.gob.mx/cibiogem/index.php/anpl/ $\underline{\text { hidalgo }}$

Trujillo JM. 2009. El ejido, símbolo de la revolución mexicana. In Luzóny JL, M Cardin eds. Problemas sociales y regionales en América Latina: estudio de casos. Barcelona, España. Universidad de Barcelona. p. 101-126. 
